\title{
Human sperm-cervical mucus interaction and the ability of spermatozoa to fuse with zona-free hamster oocytes
}

\author{
C. Barros, A. Jedlicki, I. Fuenzalida, E. Herrera, B. Arguello, P. Vigil, \\ P. Villaseca* and E. Leontic* \\ Laboratory of Embryology, Faculty of Biological Sciences and *Department of Obstetrics and \\ Gynecology, School of Medicine, Pontifical Catholic University of Chile, Casilla 114-D, Santiago, \\ Chile
}

\begin{abstract}
Summary. Samples of semen and cervical mucus were provided by 18 couples. Cervical mucus was obtained for each day possible and stored at $4^{\circ} \mathrm{C}$ until all the samples were collected. Flat capillary tubes were loaded with the mucous samples and spermatozoa from the husband's semen sample were allowed to migrate through the cervical mucus $(3 \mathrm{~cm}$ column) into culture medium. The spermatozoa recovered after migration through cervical mucus were assayed in vitro with zona-free hamster oocytes. Control experiments were carried out using spermatozoa from the same semen sample but prepared by the swimming-up technique. Altogether, 557 eggs in the control group and 1236 eggs in the experimental group were analysed, and the results demonstrated that the $\%$ of sperm penetration, the mean number of sperm decondensations per penetrated egg and the mean number of spermatozoa adhering per egg all had higher values $(P<0.05)$ for the control samples than for the experimental samples. We suggest that cervical mucus modifies human spermatozoa, as measured by their interaction with zona-free hamster oocytes.
\end{abstract}

Keywords: human; cervical mucus; gamete membrane fusion test; spermatozoa

\section{Introduction}

Human spermatozoa, while moving to the site of fertilization, must migrate through mid-cycle cervical mucus. In this process of migration the cervical mucus has been shown to have a filtering action, selecting out morphologically abnormal spermatozoa and the proportion of morphologically normal spermatozoa present in cervical mucus is higher than that of semen (Perry et al., 1977; Fredricsson \& Bjork, 1977; Hanson \& Overstreet, 1981). These findings were confirmed in an invitro study in which mid-cycle human cervical mucus was loaded in flat capillary tubes and the spermatozoa were allowed to swim through a $3-\mathrm{cm}$ mucus column into culture medium. The recovered spermatozoa were examined with the scanning electron microscope; the proportion of morphologically normal spermatozoa was significantly higher than that present in the semen sample and in the culture medium after the swim-up technique (Barros et al., 1984b). It has also been reported that spermatozoa migrating in vitro through a column of cervical mucus become aligned along the main axis of the mucus and parallel to the cervical mucus fibres (Barros et al., 1985). However, when spermatozoa are retained for several hours in cervical mucus they maintain an intact acrosome (Barros et al., 1983, 1984b) and, when the spermatozoa are treated appropriately, they can undergo the acrosome reaction and then fuse with zona-free hamster oocytes (Barros $e t$ al., 1983; Gould et al., 1984). It has not been clearly established if passage through human cervical mucus modifies the spermatozoon in its interaction with the oocyte surface as measured by the 
gamete membrane fusion test. It is also unknown whether the sperm-human cervical mucus interaction is the same throughout the different days that mid-cycle cervical mucus is present in a particular woman. Therefore, the purpose of the present work was to study the effect of spermcervical mucus interaction, after migration through cervical mucus, on the gamete membrane fusion test.

\section{Materials and Methods}

Collection of cervical mucous samples. Each woman that participated in this study was gynaecologically examined beginning on Day 10 of the cycle to determine the first day of the oestrogenic phase in which cervical mucus was present in the external os. From every women a sample of mucus was obtained on each day of the oestrogenic phase. Since it has been shown that cervical mucus can be stored for up to 14 days at $4^{\circ} \mathrm{C}$ to study sperm migration (Makler, 1976), the samples were stored in polyethylene tubes at $4^{\circ} \mathrm{C}$ until all the mucous samples of each women were collected, i.e. only one mucous sample was stored for 7 days and the rest were stored for 5 days or less.

When all the mucous samples of each women were obtained they were evaiuated according to the standard procedures of the World Health Organization (see Belsey et al., 1980) which consist of assigning values from 0 to 3 to the amount of mucus produced, to its viscosity, to its transparency, to its pattern of crystallization, and to the number of cells present in the mucous sample.

The mucus was collected as previously described by Davajan \& Kunitake (1969). This technique consists of attaching a polyethylene tube, $10-15 \mathrm{~cm}$ long and $1.4 \mathrm{~mm}$ in diameter to a $3 \mathrm{ml}$ syringe. The distal end of the tube was carefully inserted into the external os and the mucous sample was slowly drawn into the tube. Care was taken to avoid bleeding and the formation of air bubbles during this process.

Sperm migration through cervical mucus. To perform the sperm migration test, each of the cervical mucous samples of a given woman was loaded into flat $(50 \mathrm{~mm}$ long, $5 \mathrm{~mm}$ wide and $0.4 \mathrm{~mm}$ deep) capillary tubes (Vitro Dynamics, Inc, Rockaway, NJ 07866, U.S.A.): $3 \mathrm{~cm}$ were loaded with cervical mucus and $2 \mathrm{~cm}$ with TMPA (Barros et al., 1979), a culture medium containing $35 \mathrm{mg}$ human serum albumin $/ \mathrm{ml}$. The distal end, next to the TMPA culture medium, was sealed with Parafilm and the other end next to the mucous sample was placed in contact with the semen sample. The preparations were incubated for $1 \mathrm{~h}$ at $37^{\circ} \mathrm{C}$ to allow sperm migration through the cervical mucus into the culture medium. At the end of the incubation period the culture medium containing spermatozoa was removed with a fine capillary pipette and an aliquant was used to calculate the sperm concentration. The rest of the culture medium with spermatozoa was placed on a disposable tissue-culture Petri dish, covered with liquid paraffin and placed in the incubator at $37^{\circ} \mathrm{C}$. The sperm concentration recorded after migration through the cervical mucus ranged from $0.6 \times 10^{6} / \mathrm{ml}$ to $2.4 \times 10^{7} / \mathrm{ml}$.

The capillary tube with the cervical mucus was then observed with a phase-contrast microscope to determine the number of spermatozoa per field $(\times 100)$ present at the distal end of the cervical mucus column.

Preparation of spermatozoa from the semen sample by the swim-up technique. The semen samples used were those provided by the husbands with the exception of Couple 3 for whom the semen sample was provided by the man in Couple 2. A sample $(1 \mathrm{ml})$ of semen of the same semen sample as that used for the sperm migration test was mixed with $3 \mathrm{ml}$ Medium TMPA containing $4 \mathrm{mg}$ bovine serum albumin (BSA)/ml, and centrifuged for $10 \mathrm{~min}$ at $500 \mathrm{~g}$. The supernatant was discarded and the pellet was resuspended in $2 \mathrm{ml}$ of the same medium and then centrifuged for $5 \mathrm{~min}$ at $500 \mathrm{~g}$. The supernatant was discarded and the pellet was carefully covered with $0.5 \mathrm{ml}$ Medium TMPA containing $35 \mathrm{mg}$ human serum albumin $/ \mathrm{ml}$. The sample was incubated at $37^{\circ} \mathrm{C}$ for $1 \mathrm{~h}$ to allow the spermatozoa to swim up into the culture medium. The spermatozoa were then recovered from the supernatant at a concentration that ranged from $8 \times 10^{6} / \mathrm{ml}$ to $2.4 \times 10^{7} / \mathrm{ml}$.

Oocyte recovery. Mature golden hamster females were induced to superovulate by an intraperitoneal injection of 25 i.u. PMSG on the day of the post-oestrous discharge (Orsini, 1961), followed by an intraperitoneal injection of $25 \mathrm{i} . u$. hCG $60 \mathrm{~h}$ later. The females were killed $16 \mathrm{~h}$ after the hCG injection and the oviducts were dissected out and placed in a tissue-culture Petri dish (Falcon 3001). The ampullar region was broken with a pair of watchmaker's forceps and the oocytes, in cumulus, were released for treatment with a $0.1 \%$ hyaluronidase solution prepared in Medium TMPA containing $4 \mathrm{mg} \mathrm{BSA} / \mathrm{ml}$. Zona-free hamster oocytes were obtained by treating the zona-intact oocytes with $0.1 \%$ trypsin solution prepared in Medium TMPA and then thoroughly rinsed in fresh medium.

Gamete membrane fusion test. Gamete membrane fusion tests were carried out simultaneously using the spermatozoa that had migrated through all the cervical mucous samples obtained from each women (experimental) and also the spermatozoa obtained by the swim-up technique (control). The sperm concentrations for the control and experimental samples for each couple assayed were similar.

The drops of culture medium containing spermatozoa were placed in a tissue-culture Petri dish, covered with liquid paraffin and incubated at $37^{\circ} \mathrm{C}$ for $3 \mathrm{~h}$. At the end of the incubation period zona-free hamster oocytes were added to the sperm suspension and the gametes were co-incubated for 5-6h. At the end of the co-incubation period the eggs were fixed with phosphate-buffered neutral formalin and then stained with acetic orcein according to the 
method described by Toyoda \& Chang (1974). After examination by phase-contrast microscopy, the numbers of spermatozoa adhering to the oocyte surface and of sperm heads decondensed in the egg cytoplasm were recorded.

Statistical treatment of the data. For each experiment carried out with each couple the value obtained using spermatozoa that migrated through cervical mucus was subtracted from that obtained when using spermatozoa prepared by the swim-up technique. Thus, when the value of the control was higher than that of the experimental sample, the resulting value had a positive sign, while when the value of the control was lower than that of the experimental, the resulting value had a negative sign. The distribution of positive and negative values was statistically analysed using the Sign Test (Siegel, 1956): if the number of values with a positive sign departed from the expected random distribution ( 0.5 of total no. of samples), the control and experimental values were considered statistically significantly different $(P<0.05)$.

\section{Results}

In the present work 17 different semen samples were used, in which the sperm concentration and the percentage of spermatozoa with normal morphology were within the normal ranges (Table 1). The percentage of spermatozoa with progressive motility was normal in all the semen samples used except for Samples 2, 3, 5 and 10.

Cervical mucous samples were obtained from 18 different women who produced samples on different numbers of days (Table 1). Of the 53 samples, 50 had a score of $\geq 10$. When the numbers of spermatozoa still present in the cervical mucus within the flat capillary tube were evaluated, in 43 of $50(84 \%)$ samples there were $>400$ spermatozoa per field (at $\times 100$ magnification) in the most distal end of the cervical mucus, in $4(8 \%)$ samples there were $100-400$ and in $3(6 \%)$ samples there were $<100$ spermatozoa (Table 1 ).

In the gamete membrane fusion test 557 eggs were analysed for the control samples and 1236 eggs for the experimental samples. With the control samples, sperm penetration into the zona-free hamster oocytes was observed for all samples with sperm penetration values ranging from 5.4 to $100 \%$ (Table 2 ). With the experimental samples, those from $9(50 \%)$ women were able to penetrate the zona-free hamster oocytes, those from $4(22 \%)$ women (Nos 2, 5, 13, 18) were not able to penetrate and those from $5(28 \%)$ women (Nos $7,9,10,12,15)$ gave some positive and some negative results. In the experimental group the range of sperm penetration was between 4.3 and $100 \%$ (Table 2).

When the values for the experimental samples were subtracted from the results for the control samples, values with a positive sign were obtained for 45 of 53 samples $(Z=4.94 ; P<0.001)$. When the same comparison was made but using only the experimental values for which there was sperm penetration into the zona-free hamster oocytes, the difference was also significant $(Z=2 \cdot 86$; $P=0.002$ ).

For all couples except for Nos 3, 4, 9 and 16 the number of sperm decondensations was higher in the control than in the experimental samples (Table 3$)(Z=3.33 ; P<0.001)$.

To compare the number of spermatozoa adhering to the zona-free hamster oocytes in the control and in the experimental tests we chose from each group the oocytes that had been penetrated by only one spermatozoon, thus making the 2 groups more comparable. The values for control samples were all higher $(P<0.02)$ than those of the experimental samples (Table 4$)$.

\section{Discussion}

These results demonstrated that sperm passage through a column of human cervical mucus in vitro modifies the sperm surface as measured by its interaction with zona-free hamster oocytes. The results agree with previous findings (Barros et al., 1983) and with those obtained by Gould et al. (1984, 1985) and Overstreet (1986) who reported that washed sperm suspensions were more successful in attaching to and fusing with zona-free hamster oocytes than were mucus-capacitated samples. Nevertheless, we believe that although spermatozoa might achieve at least a state of 


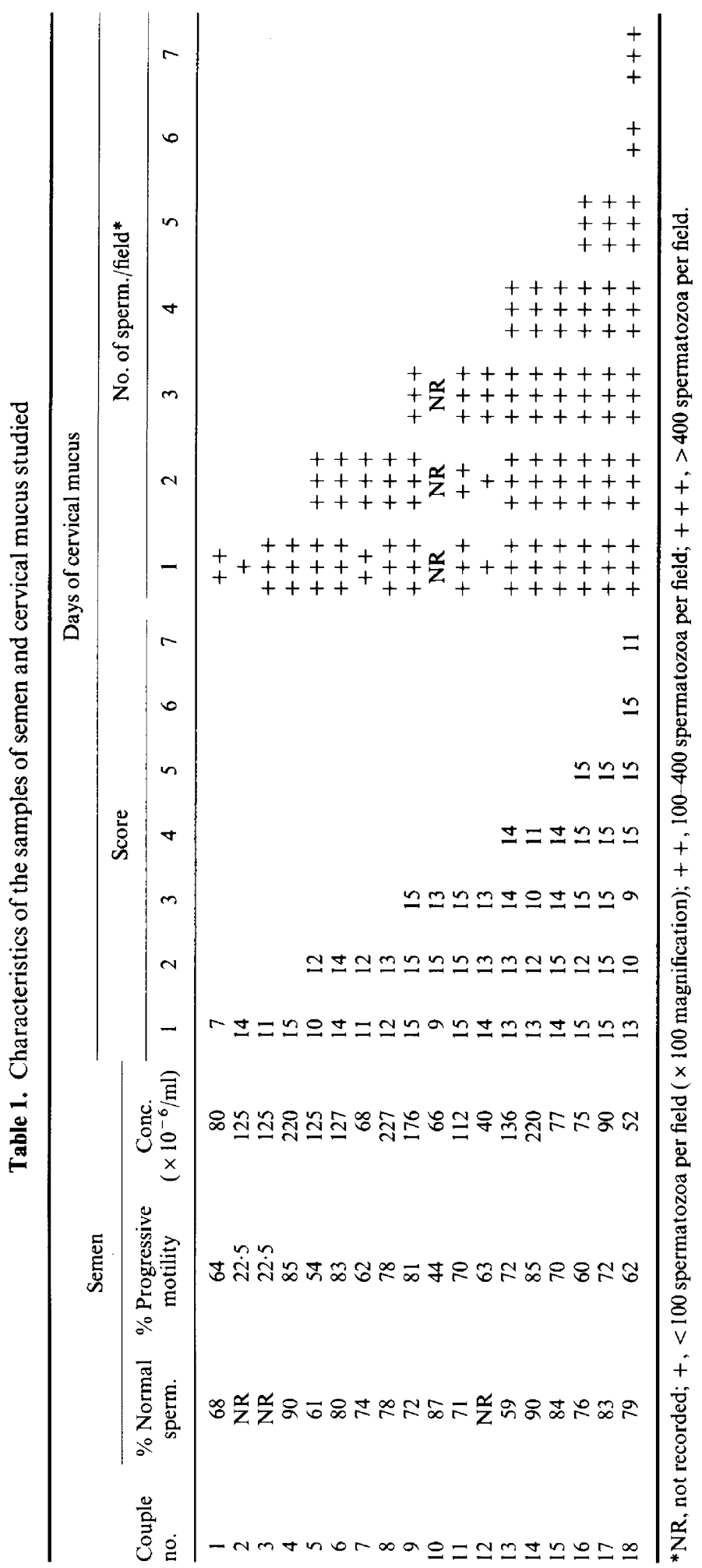




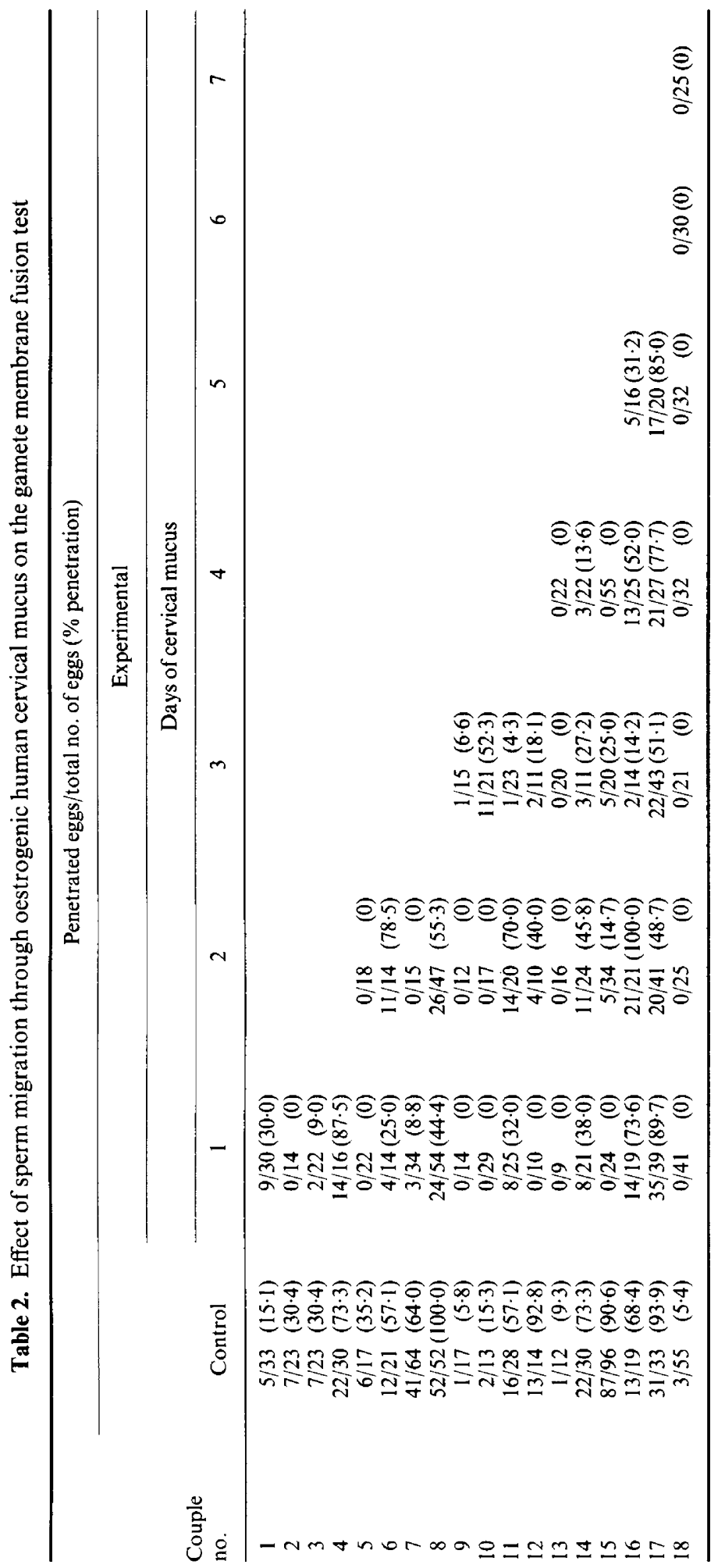


Table 3. Mean number of decondensations in penetrated eggs

\begin{tabular}{|c|c|c|c|c|c|c|}
\hline \multirow{3}{*}{$\begin{array}{l}\text { Couple } \\
\text { no.* }\end{array}$} & \multirow[b]{3}{*}{ Control } & \multicolumn{5}{|c|}{ Experimental } \\
\hline & & \multicolumn{5}{|c|}{ Days of cervical mucus } \\
\hline & & 1 & 2 & 3 & 4 & 5 \\
\hline 1 & $1 \cdot 20$ & 1.02 & & & & \\
\hline 3 & 1.00 & 1.00 & & & & \\
\hline 4 & 1.40 & 1.57 & & & & \\
\hline 6 & 1.75 & 1.45 & 1.45 & & & \\
\hline 7 & 1.60 & 1.00 & - & & & \\
\hline 8 & $2 \cdot 15$ & 1.04 & $1 \cdot 15$ & & & \\
\hline 9 & 1.00 & - & - & 1.00 & & \\
\hline 10 & $1 \cdot 50$ & - & - & 1.00 & & \\
\hline 11 & $2 \cdot 00$ & $1 \cdot 12$ & 1.28 & 1.00 & & \\
\hline 12 & $1 \cdot 38$ & - & 1.00 & 1.00 & & \\
\hline 14 & 1.40 & 1.25 & $1 \cdot 36$ & $1 \cdot 33$ & 1.00 & \\
\hline 16 & 1.61 & 2.64 & 6.90 & 1.50 & 1.84 & 1.20 \\
\hline 17 & $5 \cdot 70$ & $4 \cdot 37$ & $2 \cdot 70$ & $2 \cdot 22$ & $2 \cdot 36$ & $4 \cdot 60$ \\
\hline
\end{tabular}

${ }^{*}$ No data available for Couple 15 .

Table 4. Mean numbers of spermatozoa adhering to zona-free hamster oocytes penetrated by one spermatozoon

\begin{tabular}{|c|c|c|c|c|c|}
\hline \multirow[b]{3}{*}{ Control } & \multicolumn{5}{|c|}{ Experimental } \\
\hline & \multicolumn{5}{|c|}{ Days of cervical mucus } \\
\hline & 1 & 2 & 3 & 4 & 5 \\
\hline $13 \cdot 3$ & 0 & & & & \\
\hline $20 \cdot 0$ & 13.4 & & & & \\
\hline $50 \cdot 0$ & 0.5 & & & & \\
\hline $9 \cdot 4$ & $4 \cdot 6$ & 0.5 & & & \\
\hline $10 \cdot 1$ & $4 \cdot 8$ & $9 \cdot 7$ & & & \\
\hline $28 \cdot 6$ & $11 \cdot 5$ & $7 \cdot 1$ & & & \\
\hline $30 \cdot 0$ & $8 \cdot 3$ & $4 \cdot 2$ & & & \\
\hline $30 \cdot 3$ & $13 \cdot 5$ & $14 \cdot 8$ & $23 \cdot 0$ & $9 \cdot 5$ & $7 \cdot 6$ \\
\hline
\end{tabular}

partial capacitation while in cervical mucus they do not undergo the acrosome reaction (Barros et al., 1983). This fact is important in relation to the process of fertilization in vivo since spermatozoa displaying the acrosome reaction during passage through the cervical channel would lose or would have a reduced fertile life. In the golden hamster there is a gradual loss in the ability of spermatozoa to cross the zona pellucida as the time of sperm preincubation in a capacitating medium increases, which in turn is due to the premature occurrence of the acrosome reaction before encountering the eggs (Barros et al., 1984a).

The modification undergone by human spermatozoa after their interaction with cervical mucus proved to be so clear that a significant difference was found when comparing the percentage of penetration in the control group with all the experimental values and also when the comparison was done only with the experimental values obtained when the spermatozoa were able to fuse with the zona-free hamster oocytes. 
Our results differ from those obtained by Gould et al. (1985) with respect to the mean number of decondensations per penetrated hamster egg, since they found no difference in the number of spermatozoa present in the egg cytoplasm when using spermatozoa capacitated with cervical mucus and those capacitated with culture medium, while we found a significant difference. The higher numbers of decondensed sperm heads per penetrated egg with spermatozoa capacitated in culture medium than in cervical mucus in our study would be consistent with the other findings of the present work which showed that control spermatozoa gave higher values of sperm fusion and sperm adhesion.

Among different women there was great variability in the number of days when mucus was produced during the oestrogenic phase (1-7 days). This variation should be taken into account, for instance when performing the post-coital test, since with such variations misleading results could be obtained; that is, we believe that reliance should not be placed on only a single test and that if mucus is produced over several days several tests should also be done. In some women there was variability with regard to the values obtained for interaction with human spermatozoa in the gamete membrane fusion test; i.e. for Sample 16 (Table 2) the results of the gamete membrane fusion test ranged from 14.2 to $100 \%$ for cervical mucus obtained during the different days of the oestrogenic phase. This variation could be the result of variation in the sperm concentration, but we believe that this is unlikly because the sperm concentrations used in the present work were within the accepted range for the gamete membrane fusion test (Barros \& Jedlicki, 1985) and the sperm concentrations between the control and experimental were within the same order of magnitude. For the same couple (Couple 16) the percentage penetrations were $93 \cdot 9,89 \cdot 7,48 \cdot 7,51 \cdot 1$, 77.7 and $85 \%$ with sperm concentrations of $8 \cdot 1,22 \cdot 5,17 \cdot 5,23.7$ and $5 \times 10^{7}$ respectively. The variation in the percentage penetration of zona-free hamster oocytes by human spermatozoa after migration through cervical mucus was not related to the cervical mucus score or to the ability of the spermatozoa to migrate through cervical mucus (compare Tables 1 and 2). We suggest that the gamete membrane fusion test should be performed with spermatozoa after migration through cervical mucus, because this test would be clinically more informative, with the interaction of human spermatozoa with cervical mucus also being evaluated.

The incidence of negative results in the gamete membrane fusion test was not related to the ability of spermatozoa to migrate through cervical mucus because failures to fuse occurred with sperm counts in cervical mucus of $<100$ spermatozoa per field (Couple 2) and with $>400$ spermatozoa per field (Couple 18).

It is clear, then, that human spermatozoa can undergo certain modifications during their interaction with human cervical mucus and this interaction can have an important effect upon the process of fertilization. It would appear that the cervical mucus would prevent the spermatozoa from undergoing the acrosome reaction, thus prolonging the fertile life of the spermatozoon. It would also be apparent that the cervical mucus and the female tract in general while assuring the chances of fertilization would also reduce the probability of polyspermic fertilization.

Financed by Grants $85 / 86$ and 74/87 FIUC and from The Rockefeller Foundation. We thank FECUNDA LAB, Avda 11 de Septiembre 2155, for performing the semen and cervical mucus analyses (Table 1); Miss Ximena Lavado for secretarial assistance; and Mr Pedro Cortes for technical assistance.

\section{References}

Barros, C. \& Jedlicki, A. (1985) Human sperm fertilizing ability: Quality criteria. In Human In Vitro Fertilization: Actual Problems and Prospects, pp. 70-91. Eds J. Testart \& R. Frydman. Elsevier Science Publishers, Amsterdam.
Barros, C., Gonzalez, J., Herrera, E. \& Bustos-Obregon, E. (1979) Human sperm penetration into zona-free hamster oocytes as a test to evaluate the sperm fertilizing ability. Andrologia 11, 197-210.

Barros, C., Vigil, P., Herrera, E., Perez, A., Guadarrama, 
A. \& Bustos-Obregon, E. (1983) In vitro interaction between human spermatozoa and human cervical mucus. Micr. Electr. Biol. Cell 7, 13-19.

Barros, C., Jedlicki, A., Bize, I. \& Aguirre, E. (1984a) Relationship between the length of sperm preincubation and zona penetration in the golden hamster: A scanning electron microscopy study. Gamete Res. 9, $31-43$.

Barros, C., Vigil, P., Herrera, E., Arguello, B. \& Waker, R. (1984b) Selection of morphologically abnormal sperm by human cervical mucus. Archs Androl. (Suppl.) 12, 95-107.

Barros, C., Arguello, B., Jedlicki, A., Vigil, P. \& Herrera, E. (1985) Scanning electron microscope study of human cervical mucus. Gamete Res. 12,85-89.

Belsey, M.A., Elliason, R., Gallegos, A.J., Moghissi, K.S., Paulsen, C.A. \& Prasad, M.R.N. (1980) Laboratory Manual for the Examination of Human Semen and Semen-Cervical Mucus Interaction. Press Concern, Singapore.

Davajan, V. \& Kunitake, G.M. (1969) Fractional in vivo and in vitro examination of postcoital cervical mucus in the human. Fert. Steril. 20, 197-210.

Fredricsson, B. \& Bjork, G. (1977) Morphology of postcoital spermatozoa in the cervical secretion and its clinical significance. Fert. Steril. 28, 841-845.

Gould, J.E., Overstreet, J.W. \& Hanson, F.W. (1984) Assessment of human sperm function after recovery from the female reproductive tract. Biol. Reprod. 31, $888-894$.
Gould, J.E., Overstreet, J.W. \& Hanson, F.W. (1985) Interaction of human spermatozoa with the human zona pellucida and zona-free hamster oocyte following capacitation by exposure to human cervical mucus. Gamete Res. 12, 47-54.

Hanson, F.W. \& Overstreet, J.W. (1981) The interaction of human spermatozoa with cervical mucus in vivo. Am. J. Obstet. Gynecol. 140, 173-178.

Makler, A. (1976) A new method for evaluating cervical penetrability using daily aspirated and stored cervical mucus. Fert. Steril. 27, 533-540.

Orsini, M.W. (1961) The external vaginal phenomena characterizing the stages of the estrous cycle, pregnancy, pseudopregnancy, lactation and the anestrous hamster, Mesocricetus auratus Waterhouse. Proc. Anim. Care Panel 11, 193-206.

Overstreet, J.W. (1986) Human sperm function as assessed by fusion with zona-free hamster oocytes. Int. J. Androl. 6, 42-48.

Perry, G., Glezerman, M. \& Insler, V. (1977) Selective filtration of abnormal spermatozoa by the cervical mucus in vitro. In The Uterine Cervix in Reproduction, pp. 118-127. Eds V. Insler \& G. Bettendorf. Georg Thieme, Stuttgart.

Siegel, S. (1956) Non-Parametric Statistics for Behavioral Sciences. McGraw-Hill, New York.

Toyoda, Y. \& Chang, M.C. (1974) Fertilization of rat eggs in vitro by epididymal spermatozoa and the development of eggs following transfer. J. Reprod. Fert. 36, 9-22.

Received 21 April 1987 